DOI:

Article type: Research Paper

\title{
Ultrafast Sol-Gel Synthesis of Graphene Aerogel Materials
}

Authors: Matthew B. Lim, Matthew Hu, Sandeep Manandhar, Avery Sakshaug, Adam Strong, Leah Riley, Peter J. Pauzauskie*

Affiliations:

Matthew B. Lim, Matthew Hu, Sandeep Manandhar, Peter J. Pauzauskie

Department of Materials Science and Engineering, University of Washington, 302 Roberts

Hall, Box 352120, Seattle WA 98195-2120, USA

Avery Sakshaug, Adam Strong, Dr. Leah Riley

EnerG2 Technologies Inc., 100 NE Northlake Way, Seattle WA 98105, USA

Sandeep Manandhar

Environmental Molecular Sciences Laboratory, Pacific Northwest National Laboratory, Richland, WA

Peter J. Pauzauskie

Fundamental \& Computational Sciences Directorate, Pacific Northwest National Laboratory, Richland, WA

Keywords: graphene, aerogel, acetonitrile, resorcinol-formaldehyde, supercapacitor

\section{Abstract}

Graphene aerogels derived from graphene-oxide (GO) starting materials recently have been shown to exhibit a combination of high electrical conductivity, chemical stability, and low cost that has enabled a range of electrochemical applications. Standard synthesis protocols for manufacturing graphene aerogels require the use of sol-gel chemical reactions that are maintained at high temperatures for long periods of time ranging from 12 hours to several days. Here we report an ultrafast, acid-catalyzed sol-gel formation process in acetonitrile in which wet GO-loaded gels are realized within 2 hours at temperatures below $45^{\circ} \mathrm{C}$. Spectroscopic and electrochemical analysis following supercritical drying and pyrolysis confirms the reduction of the GO in the aerogels to $s p^{2}$ carbon crystallites with no residual carbon-nitrogen bonds from the acetonitrile or its derivatives. This rapid synthesis enhances the prospects for large-scale

* Corresponding author. Tel: 206-543-2303. E-mail: peterpz@uw.edu (Peter Pauzauskie) 
manufacturing of graphene aerogels for use in numerous applications including sorbents for environmental toxins, support materials for electrocatalysis, and high-performance electrodes for electrochemical capacitors and solar cells.

\section{Introduction}

Graphene, a two-dimensional (2D) structure of carbon atoms bonded in a hexagonal lattice, has attracted considerable attention within the scientific community for its outstanding properties, such as its electrical and thermal conductivity $\left(1738 \mathrm{~S} \mathrm{~m}^{-1}\right.$ and $\sim 5 \cdot 10^{3} \mathrm{~W} \mathrm{~m}^{-1} \mathrm{~K}^{-1}$ respectively),[1-4] intrinsic carrier mobility $\left(>2 \cdot 10^{5} \mathrm{~cm}^{2} \mathrm{~V}^{-1} \mathrm{~s}^{-1}\right),[5,6]$ theoretical surface area $\left(\sim 2600 \mathrm{~m}^{2} \mathrm{~g}^{-1}\right)$,[7] mechanical strength $(\sim 118 \mathrm{GPa})$, and elastic modulus $(\sim 1 \mathrm{TPa})$.[8] The realization of a three-dimensional (3D) network of graphene sheets would significantly expand the usability of this remarkable material. Toward this end, graphene has been integrated into bulk carbonaceous aerogel matrices that feature large surface area (400-1200 $\left.\mathrm{m}^{2} \mathrm{~g}^{-1}\right)$, high porosity $(>80 \%)$, and interconnected microstructure.[9]

Such "graphene aerogels" lend themselves to diverse potential uses that harness the ultra-porous character of aerogels in tandem with the properties of graphene. The superhydrophobicity of graphene sheets (water contact angle of $155^{\circ}$ ) makes graphene aerogel a plausible absorbent for oils, which is of interest for environmental contaminant cleanup; [10] the aerogel can furthermore be decorated with nanoparticles to target specific pollutant species, such as $\alpha-\mathrm{FeOOH}$ for arsenic removal and $\mathrm{Fe}_{3} \mathrm{O}_{4}$ for phosphate removal.[11,12] The favorable charge transport capabilities imparted by the graphene network to the aerogels makes them attractive for electrical energy-related applications as well. As electrodes for electric doublelayer capacitors (EDLCs, or "supercapacitors" for short), the conductive graphene network would not only minimize series resistance, but conceivably enhance specific capacitance, owing to graphene's extremely high surface area of $\sim 2600 \mathrm{~m}^{2} \mathrm{~g}^{-1}$ which corresponds to a theoretical capacitance as high as $\sim 550 \mathrm{~F} \mathrm{~g}^{-1}$.[4] As counter electrodes in dye-sensitized solar cells 
(DSSCs), graphene aerogel offers good catalytic activity toward the reduction of tri-iodide ions, chemical stability, high surface area yielding numerous active sites for the reduction reaction, and large pore volume enabling rapid transport of redox species.[13,14] These latter three properties also make graphene aerogel a material of interest for electrocatalyst supports for the oxygen reduction reaction (ORR) in fuel cells.

Most carbonaceous aerogels are prepared via liquid $\mathrm{CO}_{2}$-based supercritical drying and subsequent pyrolysis of wet gels resulting from the condensation of resorcinol and formaldehyde (RF). First synthesized in 1989 by Pekala and colleagues,[15] RF-derived aerogels have already been studied extensively as supercapacitor electrode materials.[16-20] Recently by Worsley et al., the pyrolysis of base $\left(\mathrm{Na}_{2} \mathrm{CO}_{3}\right)$-catalyzed RF gels loaded with graphene oxide (GO), a highly oxidized and exfoliated derivative of graphite, was shownprovided that the RF is sufficiently low in concentration - to yield extended three-dimensional graphene macroassemblies whereby the carbonized RF junctions were chemically cross-linked into the graphene framework.[21,22] These structures exhibited a bulk electrical conductivity more than two orders of magnitude higher than that of previously made 3D graphene assemblies with physical cross-links.

In spite of these results, the synthesis of base-catalyzed RF gels in water requires timeconsuming cure at elevated temperature: Zhang et al. used an overnight cure at $120^{\circ} \mathrm{C},[20]$ Worsley et al. used a $12-72$ hour cure at $85^{\circ} \mathrm{C}$,[22] while the more conventional $\mathrm{Na}_{2} \mathrm{CO}_{3}$ catalysis as introduced by Pekala stipulates even longer gelation times of up to 7 days at $85^{\circ} \mathrm{C} .[9,23]$ This could pose a major bottleneck to large-scale manufacture of RF-based graphene aerogels for commercial applications. Addressing this problem, Mulik et al. in 2007 introduced an acid $(\mathrm{HCl})$-catalyzed $\mathrm{RF}$ gelation method using acetonitrile $\left(\mathrm{CH}_{3} \mathrm{CN}\right)$ rather than water as the solvent.[23] Gelation occurred within 2 hours at room temperature and yielded aerogels chemically indistinguishable from base-catalyzed samples. 


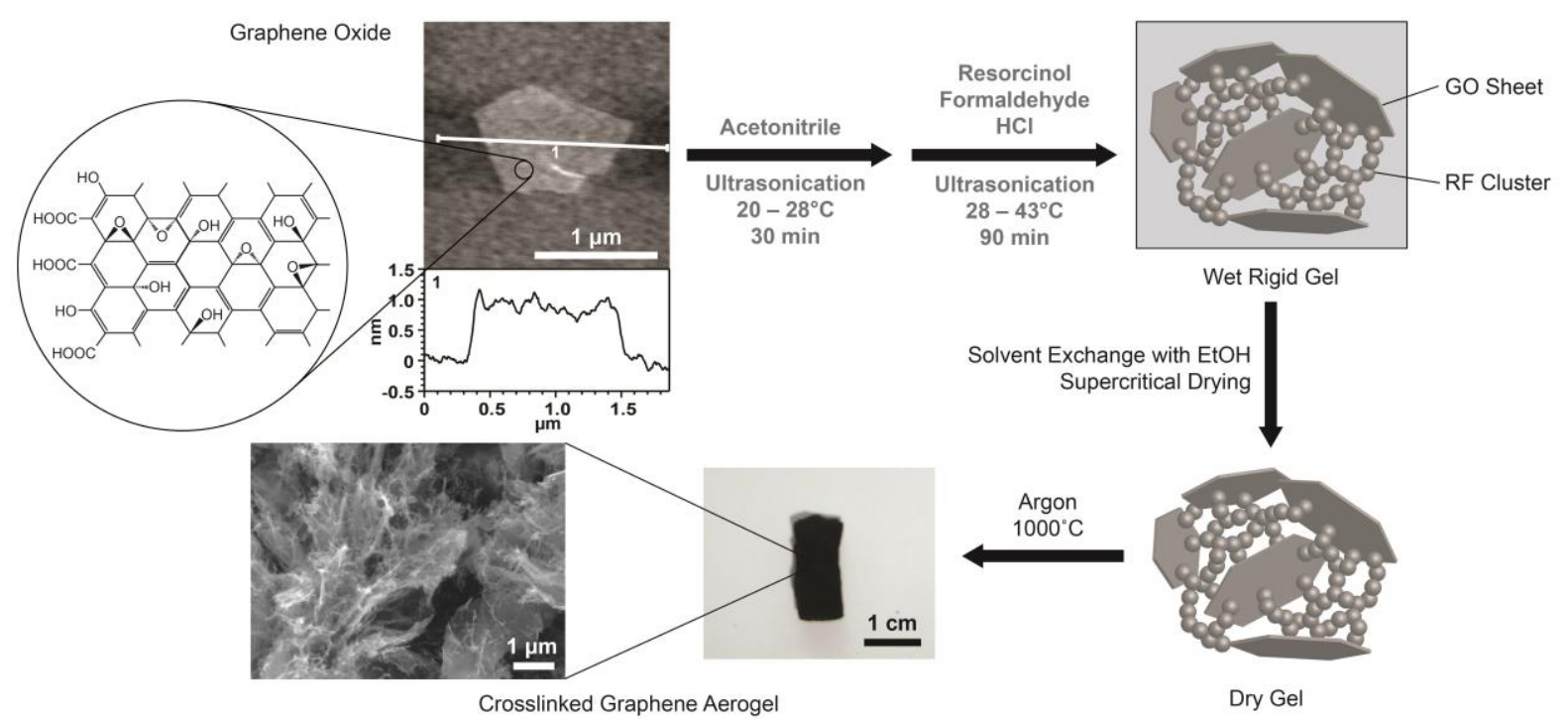

Figure 1. Diagram of the GO-RF aerogel preparation process.

Based on a previous report that GO forms a stable colloidal dispersion in acetonitrile,[24] we demonstrate in this study an acid-catalyzed route in acetonitrile whereby a mechanically rigid wet GO-RF gel can be obtained in only 2 hours at near-ambient conditions, representing a major step toward scalable and cost-effective production of graphene aerogels. The gel preparation process, outlined in Figure 1, hinges on sonication of the GO-acetonitrile dispersion, followed by a second sonication after addition of the RF-catalyst reaction mixture, to achieve the rapid gelation. Following synthesis and carbonization of both plain RF aerogels (RFA) and GO-loaded RF aerogels (GO-RFA), we characterized their microstructure and chemical composition, and also tested their performance as electrodes for coin-cell supercapacitors to illustrate a possible use case. In line with expectations of the positive effect of graphene on electrochemical properties, the carbonized GO-loaded aerogels exhibit 25\% greater areal capacitance, higher rate capability, and lower operational resistive components than their plain RF counterparts, indicating that our time-efficient synthesis of such materials could enhance the affordability of high-power energy storage. Furthermore, as the rapidity of our synthetic method also holds great promise for environmental remediation applications, where large quantities of sorbent must be obtained within a short time, we demonstrate the 
superior absorptive capabilities of our graphene aerogels, taking up more than 3 times as much cyclohexane per unit pore volume than plain RF aerogels.

a)

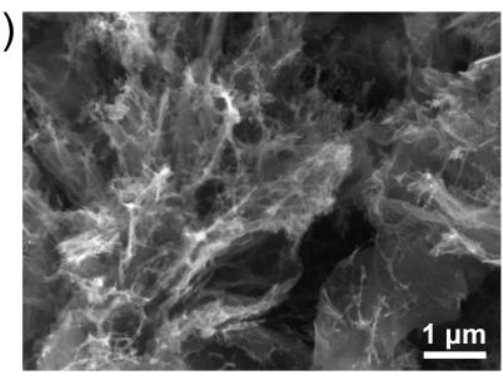

b)

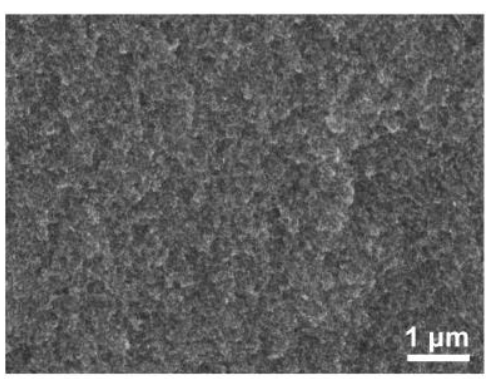

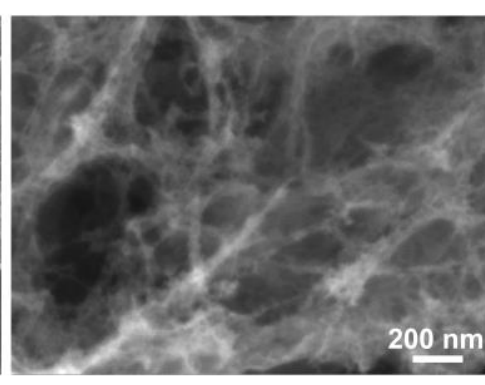
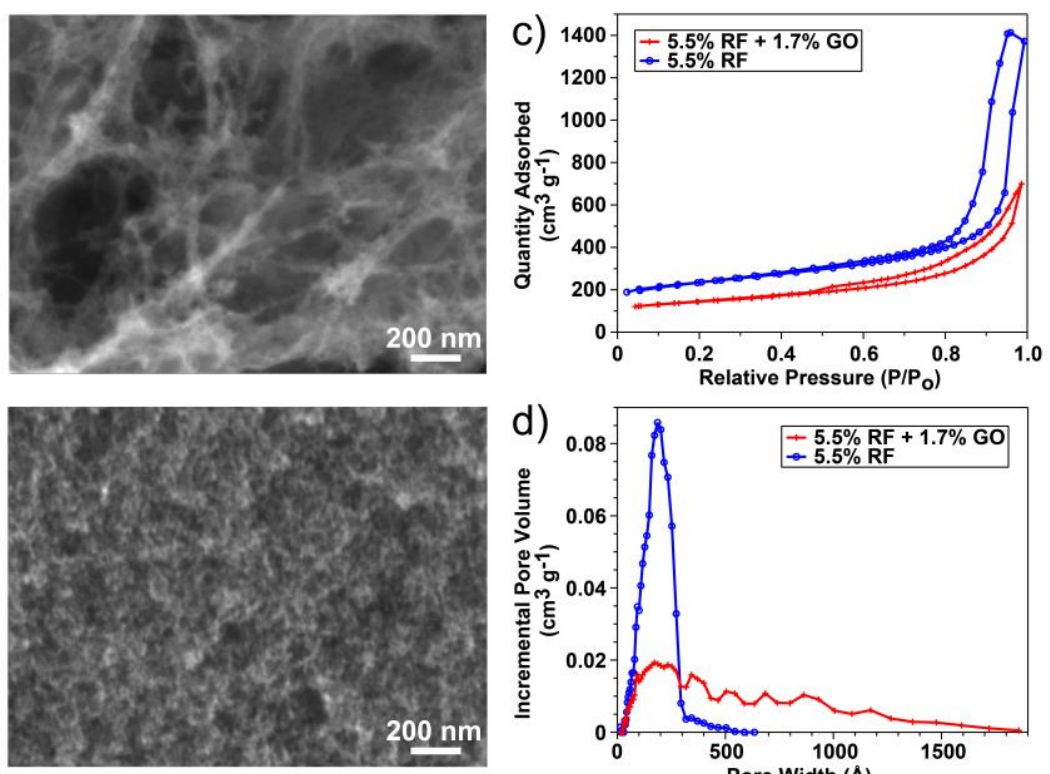

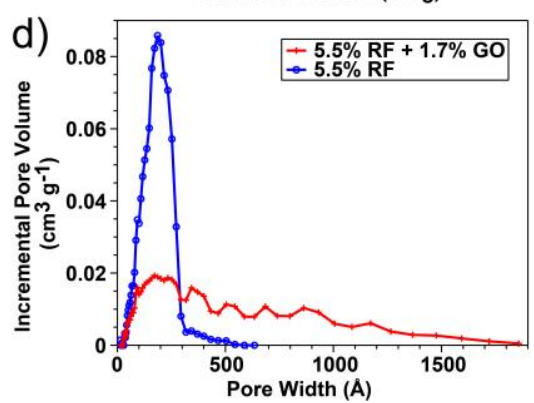

Figure 2. SEM images of the same location at different magnifications in a) pyrolyzed 5.5 wt.\% RF aerogel loaded with $1.7 \mathrm{wt} . \% \mathrm{GO}, \mathrm{b}$ ) pyrolyzed $5.5 \mathrm{wt} . \% \mathrm{RF}$ aerogel. c) Nitrogen adsorption/desorption isotherms (pressure relative to $1 \mathrm{~atm}$ ) for the aerogels. d) Non-localized density functional theory (NLDFT) pore size distribution plots for the aerogels.

\section{Experimental}

\subsection{Aerogel Synthesis}

For the GO-RFA, commercially obtained multilayer GO (Cheap Tubes Inc., 2-4 layers, total thickness $<3 \mathrm{~nm}$ ) was suspended in acetonitrile (AN, EMD Millipore) in the desired concentration and dispersed by sonication at ambient conditions for 30 minutes using a Branson 1510R-DTH ultrasonic cleaner (sonic power $70 \mathrm{~W}$, frequency $\sim 42 \mathrm{kHz}$ ). (For the RFA, this step was omitted.) Although the sonicator was initially at room temperature and no heat was applied, the energy of the ultrasonic waves caused the bath to heat up gradually from $20^{\circ} \mathrm{C}$ to $28^{\circ} \mathrm{C}$ over this time interval. Following the work of Mulik et al.,[23] resorcinol (R, SigmaAldrich), formaldehyde (F, Sigma-Aldrich, 37\% w/w aqueous solution methanol-stabilized), and hydrochloric acid catalyst $(\mathrm{C}$, Macron, $37 \% \mathrm{w} / \mathrm{w})$ were added to the $\mathrm{GO}$ suspension to achieve the weight ratio $\mathrm{R}: \mathrm{GO}=2: 1$ and the following molar ratios: $\mathrm{R}: \mathrm{F}=1: 2, \mathrm{R}: \mathrm{C}=8.4: 1$, $\mathrm{R}: \mathrm{AN}=1: 76$ which corresponds to $5.5 \mathrm{wt} \% \mathrm{RF}$ and $1.7 \mathrm{wt} \% \mathrm{GO}$. The mixture was further 
sonicated, with a solid gel forming within 30 minutes, at which time the temperature of the bath had risen to $35^{\circ} \mathrm{C}$. The wet gel was maintained under sonication for an additional hour, with the bath temperature rising to $43^{\circ} \mathrm{C}$ in this time, to increase the mechanical rigidity of the gel before extracting it from the reaction vessel. The gel was then washed with ethanol (4 times over 48 hours) to remove all acetonitrile from the pores of the gel network, and dried in supercritical $\mathrm{CO}_{2}$ in an autoclave (E3100, Quorum Technologies). Supercritical drying was used for the gel in order to prevent collapse of the solid matrix and keep the porous structure intact. The dried gel was subsequently pyrolyzed under flowing argon gas $(0.4 \mathrm{~L} / \mathrm{min})$ at $1000^{\circ} \mathrm{C}$ for 4 hours in a Thermo Scientific Lindberg/Blue M tube furnace.

\subsection{Electrode and Coin Cell Fabrication}

In collaboration with EnerG2 Technologies, Inc., the pyrolyzed GO-RFA and pyrolyzed RFA (GO-RFA-P and RFA-P) material was processed into coin cell electrodes for electrochemical testing. Briefly, a mixture of $70 \mathrm{wt} \%$ aerogel, $20 \mathrm{wt} \%$ PTFE tape (binder), and $10 \mathrm{wt} \%$ SUPER P conductive carbon additive (Timcal) was ground together in a mortar and pestle, with acetone to aid dispersion. The resulting sheet of electrode was then repeatedly flattened with a glass rolling pin to a thickness of $\sim 150 \mu \mathrm{m}$, punched to $0.5 \mathrm{inch}$, and dried in vacuum at $195^{\circ} \mathrm{C}$ for 8 hours. Supercapacitor devices were assembled with CR2325 coin cell casings (National Research Council Canada) in an argon-filled glove box. A symmetric two-electrode configuration was employed, with rayon separator (Nippon Kodoshi Corporation) and tetraethylammonium tetrafluoroborate (TEABF4) electrolyte (1 M in acetonitrile).

\subsection{Microstructural Characterization}

Nitrogen sorption isotherms of the GO-RFA-P and RFA-P were collected at $77 \mathrm{~K}$ in the range of relative pressures of $\mathrm{P} / \mathrm{P}_{0}=0.01-1$ using a Micromeritics TriStar II 3020 analyzer. To ensure removal of pre-adsorbed species, samples were heated in vacuum to $400^{\circ} \mathrm{C}$ for at least 8 hours 
prior to analysis. Surface area and pore size measurements were performed by the BrunauerEmmett-Teller (BET) and non-localized density functional theory (NLDFT) methods, respectively, using Micromeritics DataMaster software. The relative pressure range of $\mathrm{P} / \mathrm{P}_{0}=$ 0.03-0.11 was used for multipoint surface area calculations. The surface morphology of the aerogels was investigated using a FEI Sirion field-emission scanning electron microscope (SEM) with $5 \mathrm{kV}$ accelerating voltage. X-ray diffraction (XRD) measurements were performed on flat sections of the bulk GO-RFA-P that were affixed to a $<100>$ silicon wafer substrate with double-sided tape. The measurement system was a Bruker D8 Discover X-ray diffractometer equipped with a General Area Detector Diffraction System (GADDS) using a $\mathrm{Cu} \mathrm{K}_{\alpha}$ radiation source $\left(\lambda_{\mathrm{K} \alpha}=1.54 \AA\right)$. Measurements were conducted over $2 \theta=10^{\circ}$ to $60^{\circ}$ with step size $0.05^{\circ}$.

\subsection{Chemical Characterization}

FTIR spectroscopy of the aerogels was performed on a Bruker VERTEX 70 Spectrometer using the $\mathrm{KBr}$ pellet in transmission mode. The IR spectrum of GO was obtained by the attenuated total reflectance (ATR) technique using the same spectrometer with an integrated Bruker PLATINUM ATR accessory (Unit A225/Q1). Raman spectroscopy was performed using 532 $\mathrm{nm}(2.33 \mathrm{eV})$ laser excitation, with the back-scattered light being analyzed with an Acton Research Corporation SpectraPro-500i liquid nitrogen-cooled spectrometer. For electrochemical tests, the aerogel coin cells were conditioned on a Bio-Logic VMP3 potentiostat/galvanostat over 5 cycles to ensure complete electrolyte permeation, followed by 6 galvanostatic charge-discharge cycles at 1, 5, 10,25, 50, and $100 \mathrm{~mA}$. This corresponds to current densities of $0.078,0.39,0.78,1.95,3.90$, and $7.78 \mathrm{~A} \mathrm{~g}^{-1}$ for the GO-RFA-P and 0.067, $0.34,0.67,1.68,3.36$, and $6.71 \mathrm{~A} \mathrm{~g}^{-1}$ for the RFA-P. The discharge curves were used to interpolate electrode capacitance at $0.5 \mathrm{~A} \mathrm{~g}^{-1}$. Immediately following rate testing, electrochemical impedance spectroscopy was conducted at $2.0 \mathrm{~V}$ using $10 \mathrm{mV}$ sinusoidal 
amplitude between $400 \mathrm{kHz}-10 \mathrm{mHz}$. Cyclic voltammetry was then performed, sweeping between 0 and $2.7 \mathrm{~V}$ at $20 \mathrm{mV} \mathrm{s}^{-1}$.

\subsection{Absorption of Cyclohexane by the Pyrolyzed Aerogels}

The absorptive capabilities of the GO-RFA-P and RFA-P for non-polar liquids was tested using cyclohexane as an example solvent. A small (10-20 mg) piece of aerogel was weighed, immersed in cyclohexane for approximately $10 \mathrm{~min}$, and then weighed again. The wet mass measurement was performed quickly to minimize the effect of solvent evaporation. The weight gain of each aerogel was taken as the average of three measurements.

\section{Results and Discussion}

\subsection{Microstructural Analysis of Pyrolyzed Aerogels}

Scanning electron microscope (SEM) images of the GO-RFA-P (Figure 2a) revealed an assembly of randomly oriented, presumably graphitic or graphenic sheets, on the order of several microns across, embedded in a network of carbonized chains of RF condensate. The spacing between neighboring carbonized RF chains is large, in many cases over $100 \mathrm{~nm}$ across. In addition, the network is finer on the surface of the graphitic sheets than in the bulk of the aerogel. Smaller RF particles are formed on the surface of GO sheets than in the bulk solvent because the presence of negatively charged $\mathrm{O}^{-}$and $\mathrm{COO}^{-}$groups on the $\mathrm{GO}$ surface locally accelerates the deprotonation of resorcinol, which has been reported to be the first step in the RF polymerization mechanism.[25] In contrast to the GO-RFA-P, images of the RFA-P without GO reveal a sponge-like rather than filamentous morphology (Figure 2b). The RF network is more evenly dense with finer pores and features. At higher magnification, the RFA$\mathrm{P}$ appears to consist of numerous small, uniformly sized $(<20 \mathrm{~nm})$ beadlike particles joined in a disordered array, interspersed with similarly sized voids. 
Nitrogen sorption isotherms, from which pore structure and surface area can be determined, are plotted in Figure $2 \mathrm{c}$ for the RFA-P and GO-RFA-P. The shapes of the hysteresis loop for the two aerogels agree with their microstructural features as observed with SEM. The Type H4 hysteresis loop (IUPAC classification) in the sorption isotherm of the GORFA-P is commonly associated with slit-shaped pores or aggregates of plate-like particles, the latter of which is representative of the incorporated GO.[26] The sorption isotherm of the RFAP shows Type $\mathrm{H} 1$ hysteresis, which is characteristic of materials having well-defined cylindrical pores or spheroidal particles of approximately the same size.[26] Additionally, both total adsorption and the size of the hysteresis loop for the GO-RFA-P were smaller than that of the RFA-P, suggesting that the GO-RFA-P has lower surface area. In support of this claim, the surface areas as calculated by the Brunauer-Emmett-Teller (BET) method were $542 \mathrm{~m}^{2} \mathrm{~g}^{-1}$ and $745 \mathrm{~m}^{2} \mathrm{~g}^{-1}$ for the GO-RFA-P and the RFA-P, respectively; furthermore, the average pore volume was $1.43 \mathrm{~cm}^{3} \mathrm{~g}^{-1}$ for the RFA-P, compared to $0.959 \mathrm{~cm}^{3} \mathrm{~g}^{-1}$ for the GO-RFA-P.

The low surface area of the GO-RFA-P compared to the theoretical surface area of a single graphene sheet $\left(>2600 \mathrm{~m}^{2} \mathrm{~g}^{-1}\right)$ [27] may be due to layering or overlapping of graphene sheets within the aerogel. The multilayer GO, used as the initial loading material, retains its layered structure even after addition to the RF, causing the low overall surface area. It is expected that single layered GO would yield a GO-RFA-P surface area closer to $2600 \mathrm{~m}^{2} \mathrm{~g}^{-1}$. The slightly lower surface area of the GO-RFA-P compared to the RFA-P is reflective of the broader pore size distribution of the former (Figure 2d). Much of the pore volume of the RFAP is confined to pores of $0-300 \AA$ wide, with a peak width of $186 \AA$, and no pores of greater width than about $550 \AA$. The GO-RFA-P, while having a similar peak pore width $(172 \AA)$, exhibits a tail in its distribution that is more than four times longer, spanning from the peak pore width to $1850 \AA$. This is consistent with our SEM observation of a less dense RF network in the GO-RFA-P. Additionally, the prevalence of macropores in the GO-RFA-P can be corroborated with an earlier study on unpyrolyzed GO-RF gels by Guo et al., who noted an 
increase in RF particle size to $>50 \mathrm{~nm}$ as well as a lessening of RF polymer formation within the bulk liquid as GO loading was increased to a level (2 wt\%) similar to our aerogels.[25]

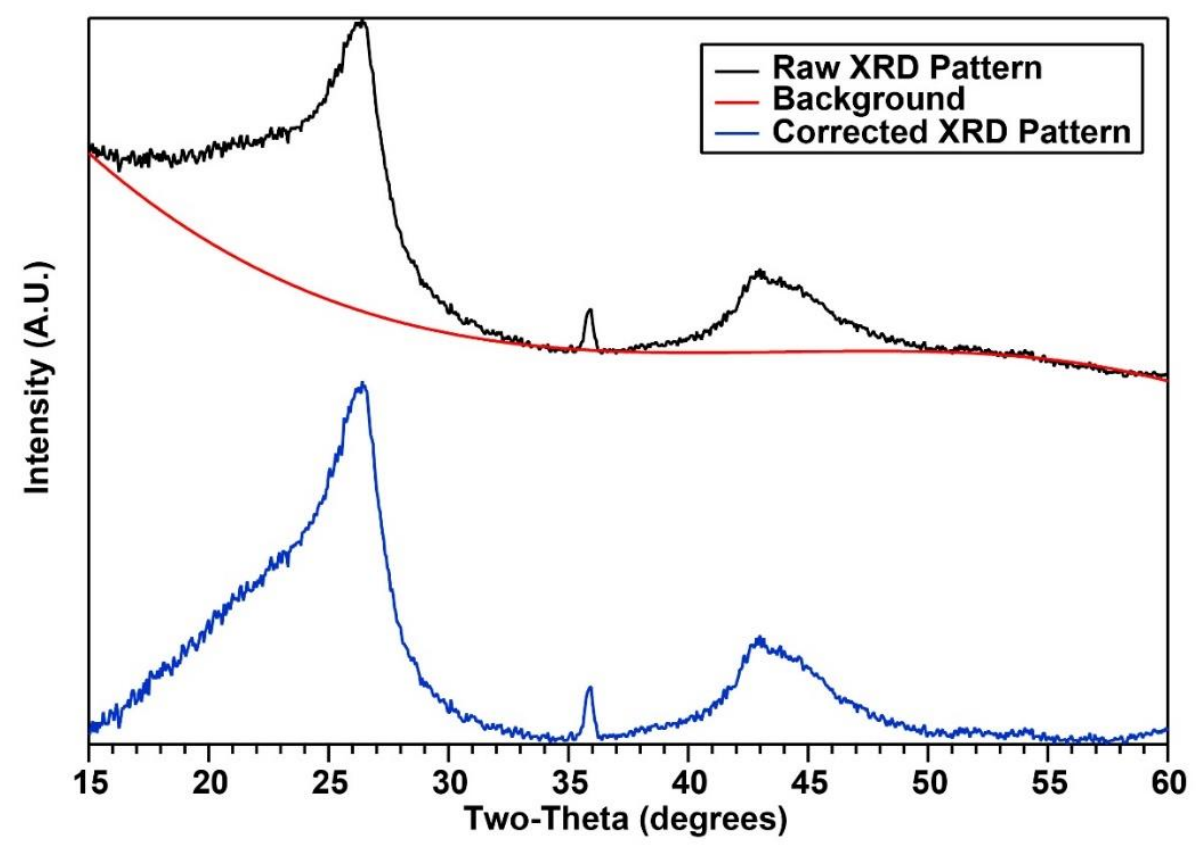

Figure 3. (Top) Raw X-ray diffractogram of the bulk pyrolyzed GO-RFA with 5.5 wt $\%$ RF + $1.7 \mathrm{wt} \%$ GO. (Middle) Polynomial curve representing presumed background of raw XRD signal. (Bottom) X-ray diffractogram with background subtracted.

The graphitization of the GO during pyrolysis is confirmed by the X-ray diffraction pattern of GO-RFA-P (Figure 3). The intense diffraction peak at $2 \theta=26.4^{\circ}$ is that of the $(002)$ planes (basal planes) of graphite, while the weaker, broader peak centered on $2 \theta=44^{\circ}$ originates from the (101) planes. A third weak but sharp peak at $2 \theta=36.0^{\circ}$ suggests the presence of a small amount of beta silicon carbide ( $\beta$-SiC), whose intense (111) diffraction peak occurs at this angle.[28] This likely originated from our use of a quartz tube furnace for GO-RFA pyrolysis. At temperatures of $1000^{\circ} \mathrm{C}$ or greater, silica undergoes a solid-state reaction with carbon to form silicon carbide and carbon monoxide.[29,30]

The size of the graphitic crystallites in the aerogel can be quantified from the (002) graphitic peak of the background-subtracted XRD pattern using the Scherrer equation:

$$
L_{a}=\frac{1.84 \lambda_{K \alpha}}{\beta \cos \theta}
$$


where $L_{a}$ is the apparent crystallite size perpendicular to the $c$-axis (i.e., in-plane), $\theta$ is the position of the (002) peak $\left(13.2^{\circ}\right)$, and $\beta$ is the full width at half-maximum (FWHM) of the (002) peak in $2 \theta$ (radians) units.[31] The $\beta$ parameter was corrected for instrumental broadening using the expression $\beta=\sqrt{\beta_{m}^{2}-\beta_{S i}^{2}}$, where $\beta_{m}$ is the measured FWHM of the (002) peak for the aerogel and $\beta_{S i}$ is the FWHM of the (111) peak of a standard single-crystal silicon wafer, which was obtained experimentally. This silicon peak was chosen since it occurs at a $2 \theta$ value $\left(28.5^{\circ}\right)$ very close to the (002) graphitic peak. The crystallite size calculated by this method was $3.5 \mathrm{~nm}$. This is within the same order of magnitude as the crystallite size calculated from the Raman spectrograph of the aerogel $(9.9 \mathrm{~nm}$, see Section 3.2) and therefore suggests that the micron-scale sheets observed in Figure 2a are polycrystalline.

\subsection{Spectroscopic Analysis of Chemical Bonding in Aerogels Pre-and Post-Pyrolysis}
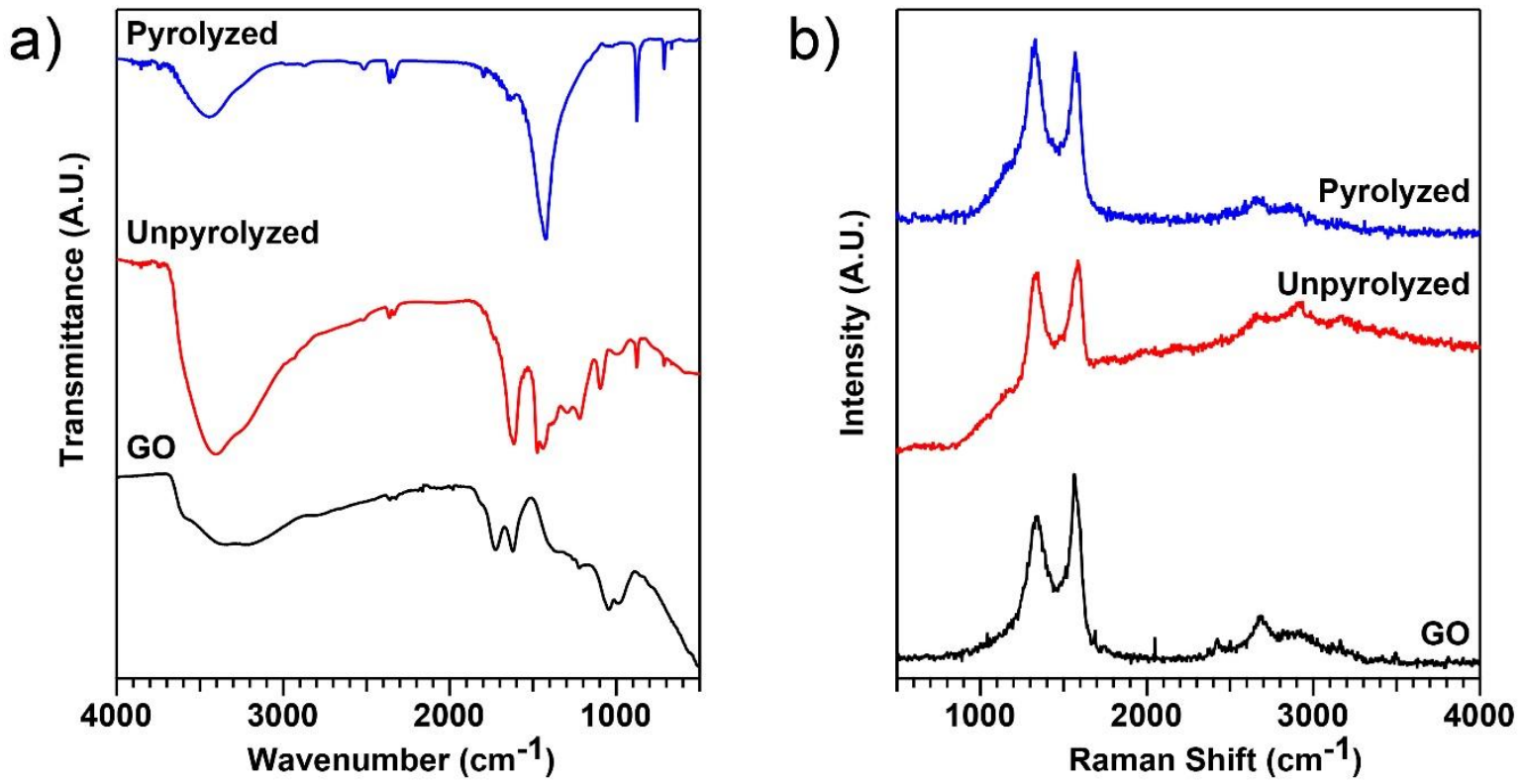

Figure 4. Normalized a) FTIR spectra and b) Raman spectra of the pyrolyzed and unpyrolyzed GO-RFA (5.5 wt\% RF + $1.7 \mathrm{wt} \% \mathrm{GO})$. GO is included for reference.

Vibrational modes present in the bonds of the GO-RFA were investigated with Fourier transform infrared (FTIR) spectroscopy and Raman spectroscopy (Figure 4). IR spectra of GO reference powder and of the GO-RFA before pyrolysis (GO-RFA-U) and after pyrolysis are 
presented in Figure 4a. Characteristic absorbances were assigned from the literature.[32] The two small bands at 2360 and $2340 \mathrm{~cm}^{-1}$ do not originate in the analyzed material, but correspond to residual carbon dioxide from measurement conditions. Most importantly, comparison of the spectra of the two aerogels confirms the thermal decomposition of the oxygen-containing functional groups in GO. The absorption bands of the C-O-C stretch of epoxy groups at 1221 $\mathrm{cm}^{-1}$, and of the C-O stretch of alkoxy groups at $1095 \mathrm{~cm}^{-1}$, appear in the spectrum of GO-RFAU but not of GO-RFA-P. Specifically, the peaks of the C-O stretch at $1095 \mathrm{~cm}^{-1}$ and the C-H bend at $1470 \mathrm{~cm}^{-1}$ in the GO-RFA-U are associated with the methylene ether bridges and methylene bridges, respectively, that form between aromatic rings in the RF condensate.[15,33,34] The loss of carbonyl groups was also observed; both GO powder and GO-RFA-U display a medium-intensity absorption band of the carboxylic acid $\mathrm{C}=\mathrm{O}$ stretch around $1730 \mathrm{~cm}^{-1}$ (though only appearing as a shoulder in the latter due to convolution with a more intense adjacent band), while the corresponding band in GO-RFA-P (again convoluted) is significantly weaker.

Interestingly, both GO-RFA-U and GO-RFA-P, in addition to GO powder, display the broad absorption band starting at $3700 \mathrm{~cm}^{-1}$ of the hydrogen bonded $\mathrm{O}-\mathrm{H}$ stretch. However, in the GO and GO-RFA-U, this band stretches all the way to $2500 \mathrm{~cm}^{-1}$, encompassing the characteristic O-H absorption frequencies of both alcohols $\left(3600-3000 \mathrm{~cm}^{-1}\right)$ and carboxylic acids (3000-2500 $\mathrm{cm}^{-1}$ ), while in GO-RFA-P the band only goes to $3050 \mathrm{~cm}^{-1}$; furthermore, this $\mathrm{O}-\mathrm{H}$ absorption band is significantly more intense in GO-RFA-U than in GO-RFA-P. These observations suggest that pyrolysis resulted in near-complete removal of the carboxylic acids that are abundant on the edge planes of pristine GO (see Figure 1) and partial removal of the alcohols. This conclusion is consistent with more detailed investigations of the structural evolution of GO during thermal reduction, which have established that the carboxyl groups become highly unstable at temperatures above $400^{\circ} \mathrm{C}$.[35] The continued existence of the alcohol $\mathrm{O}-\mathrm{H}$ band in the carbonized gel can be explained by the reaction of basal plane epoxides 
around $400^{\circ} \mathrm{C}$ with adjacent hydroxyl groups to form phenol groups, which have been shown to survive even at $1000^{\circ} \mathrm{C},[35]$ the temperature at which our GO-RFA was pyrolyzed. It is thermodynamically difficult to completely pyrolyze $\mathrm{C}-\mathrm{OH}$, mainly because of its position within the interlayer spacing between intact conjugated domains.[36,37]

The presence of aromatic rings in both the unpyrolyzed and pyrolyzed GO-RFA, originating from the $\mathrm{GO}$ and $\mathrm{RF}$ condensate, is evident from the aromatic $\mathrm{C}=\mathrm{C}$ stretching bands: an intense band at $1425 \mathrm{~cm}^{-1}$ and much weaker band at $1630 \mathrm{~cm}^{-1}$ for the GO-RFA-P; the two sharp bands at $1440 \mathrm{~cm}^{-1}$ and $1615 \mathrm{~cm}^{-1}$ for the GO-RFA-U; and a single sharp band at 1620 $\mathrm{cm}^{-1}$ for the GO. The occurrence of only a single peak in GO for this type of vibration may be a result of convolution, as suggested by the steady drop in transmission from $1500 \mathrm{~cm}^{-1}$ to 1400 $\mathrm{cm}^{-1}$, the range where the second peak was observed in the two aerogel samples. The slight shift of the $\mathrm{C}=\mathrm{C}$ bands following pyrolysis may reflect the accompanying reduction in the extent of aromatic substitution in the material as well as the change in nature of the substituents, from epoxy, carboxyl, and phenol groups to phenol groups alone. Interestingly, the sharp aromatic C-H out-of-plane bending bands visible at 879,712 , and $667 \mathrm{~cm}^{-1}$ in GO-RFA-U appear to be at similar positions in GO-RFA-P, indicating that the substitution pattern remains largely the same.[38,39] There are no observable absorption bands attributable to $\mathrm{C} \equiv \mathrm{N}\left(2260-2240 \mathrm{~cm}^{-1}\right)$, $\mathrm{C}=\mathrm{N}\left(1690-1650 \mathrm{~cm}^{-1}\right)$, or $\mathrm{C}-\mathrm{N}\left(1350-1000 \mathrm{~cm}^{-1}\right)$ in the recovered GO-loaded aerogel following pyrolysis, suggesting complete removal of acetonitrile and any nitrogen-containing species derived from acetonitrile.

Raman spectra of the GO-RFA before and after pyrolysis are shown in Figure 4b. The peak centered at about $1570 \mathrm{~cm}^{-1}$ denotes the $\mathrm{G}$ band which is associated with the doubly degenerate (iTO and LO) phonon mode with $\mathrm{E}_{2 \mathrm{~g}}$ symmetry at the Brillouin zone center.[40] The peak at $1330 \mathrm{~cm}^{-1}$ is the disorder-induced $\mathrm{D}$ band, attributed to $\mathrm{A}_{1 \mathrm{~g}}$ modes at the zone boundary that consist only of C-C bond stretching motions.[41] The ratio of the integrated intensities of the two bands, $\mathrm{I}_{\mathrm{D}} / \mathrm{I}_{\mathrm{G}}$, has been reported to be inversely proportional to the in- 
plane size $\mathrm{L}_{\mathrm{a}}$ of the graphitic $s p^{2}$ crystallites in the sample;[42] a higher intensity of the $\mathrm{D}$ band indicates a higher fraction of disordered amorphous carbon.[43,44] More specifically, the crystallite size can be approximated by

$L_{a}(\mathrm{~nm})=\frac{560}{E_{l}^{4}}\left(\frac{I_{D}}{I_{G}}\right)^{-1}$

where $E_{l}$ is the excitation laser energy in eV.[45] For our Raman analysis, $E_{l}=2.33 \mathrm{eV}(\lambda=$ $532 \mathrm{~nm}), I_{D} / I_{G}$ is calculated to be 1.56 for GO-RFA-U and 1.92 for GO-RFA-P, corresponding to $L_{a}=12.2 \mathrm{~nm}$ and $9.9 \mathrm{~nm}$ respectively. The latter is on the same order of magnitude as the crystallite size of $3.5 \mathrm{~nm}$ obtained from the XRD data of GO-RFA-P (see Section 3.1). The slight decrease in the size of the graphitic domains following pyrolysis may be attributed to breakage of chemical cross-links between GO sheets that were originally formed during gelation, in the same manner that breakage of methylene and methylene ether bridges between individual aromatic rings has been observed for pyrolysis of pure RF gels.[46] This would leave the sheets with dangling bonds that could then form interplanar $s p^{3}$ bridges as described by Milev et al.,[47] reducing the extent of each individual graphitic domain. 
a)
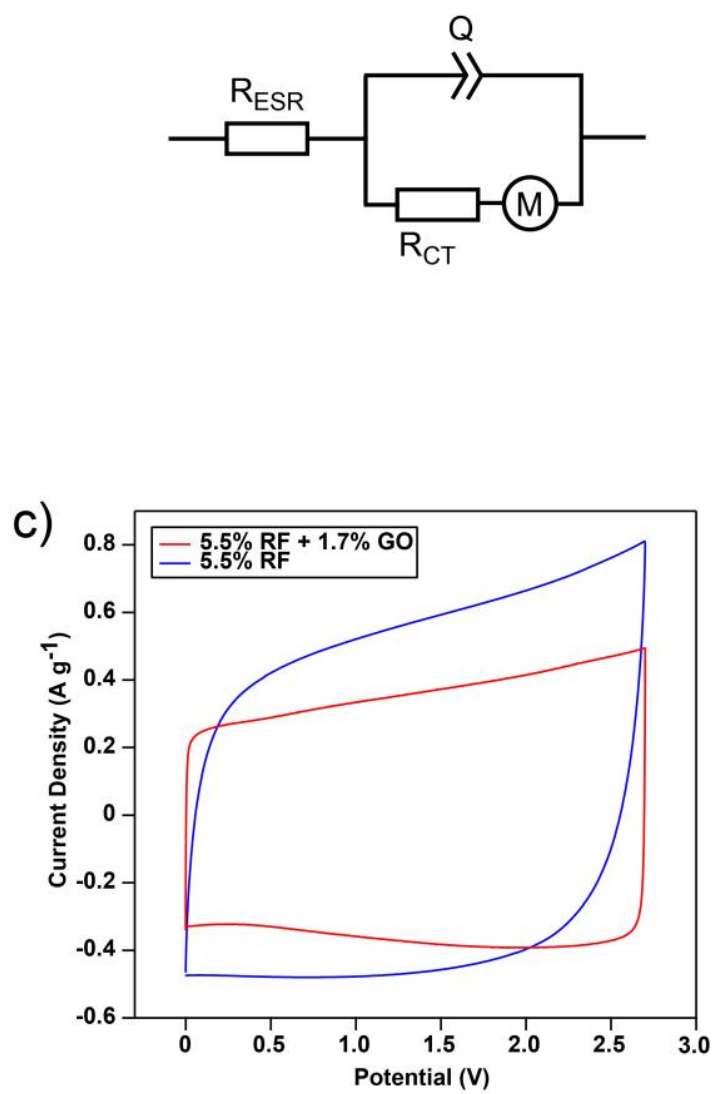

b)

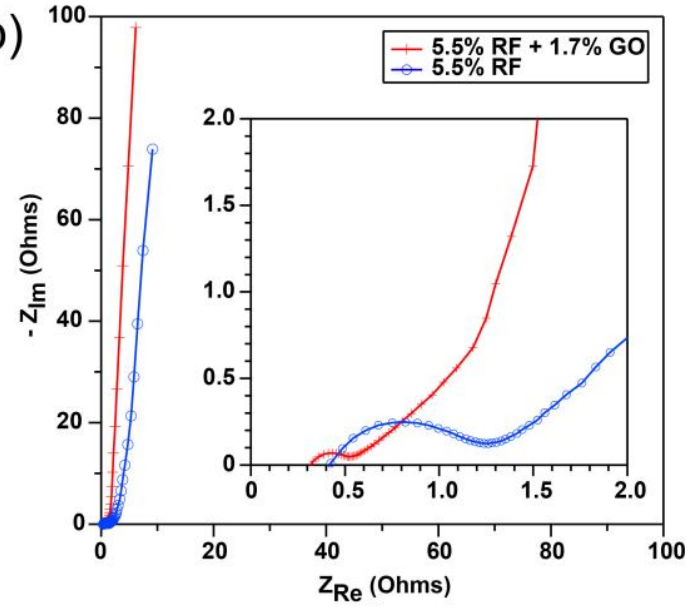

d)

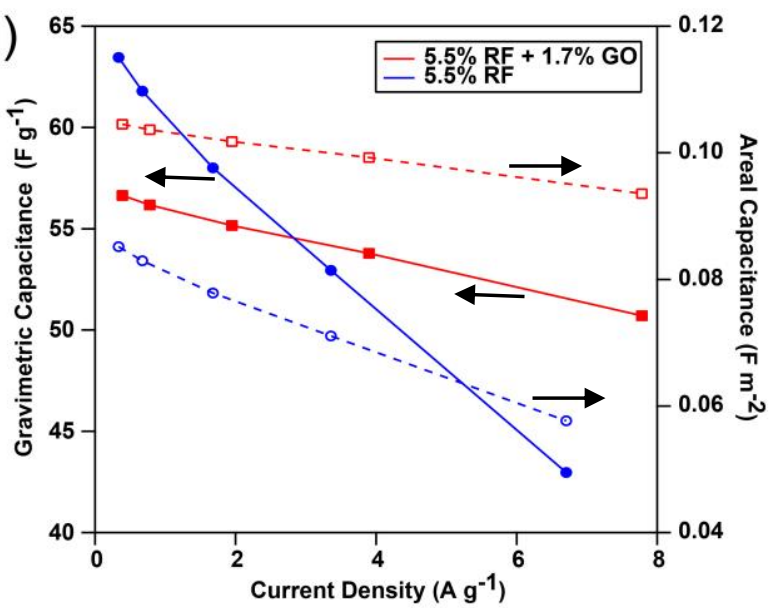

Figure 5. Coin cell testing results of the pyrolyzed GO-loaded RF aerogels and plain RF aerogels. a) Equivalent circuit diagram of the supercapacitor. b) EIS Nyquist plots (inset shows more detail of high to mid frequency range). c) Cyclic voltammograms (sweep rate $20 \mathrm{mV} \mathrm{s}^{-1}$ ). d) Rate capability plot obtained from galvanostatic charge-discharge tests. Solid lines show trend in gravimetric capacitance while dashed lines show trend in areal capacitance.

\subsection{Electrochemical Testing of Pyrolyzed Aerogels as Supercapacitor Electrodes}

The capacitances of the as-assembled pyrolyzed GO-RFA and RFA supercapacitor cells were evaluated by galvanostatic charge-discharge tests between 0.1 and $2.7 \mathrm{~V}$ at increasing current densities corresponding to applied currents of 5, 10,25, 50, and $100 \mathrm{~mA}$. The specific capacitances for a single electrode at each current density were calculated from the resulting discharge curves (Supporting Information, Figure S3) according to the following equation:

$C_{s}=\frac{4 I \Delta t}{m \Delta V}$

where $C_{s}$ is the specific capacitance, $I$ is the constant discharge current, $m$ is the mass of active material, $\Delta V$ is the potential window, and $\Delta t$ is the time elapsed in the range of $\Delta V . C_{s}$ at $0.5 \mathrm{~A}$ 
$\mathrm{g}^{-1}$ was interpolated from a parabolic fit of the $C_{s}$ versus current density data, where the parabolic fit accounts for the increasing effect of leakage current on the calculated capacitance at lower applied currents. (The $1 \mathrm{~mA}$ discharge data was excluded from the fit for this same reason.) The GO-RFA-P supercapacitor exhibited a gravimetric specific capacitance of $56.3 \mathrm{~F}$ $\mathrm{g}^{-1}$ at $0.5 \mathrm{~A} \mathrm{~g}^{-1}$, which is similar to that of the RFA-P supercapacitor at the same current density $\left(61.8 \mathrm{~F} \mathrm{~g}^{-1}\right)$. However, due to the RFA-P's larger surface area, the performance improvement using GO-RFA-P becomes apparent when capacitance is expressed per unit area $\left(0.104 \mathrm{~F} \mathrm{~m}^{-2}\right.$ for GO-RFA-P versus $0.0830 \mathrm{~F} \mathrm{~m}^{-2}$ for RFA-P).

Resistance is a key property of performance in assessing the viability of GO-RFA-P for supercapacitor applications. Since supercapacitors are designed for high power, manufacturers look for materials that can minimize system resistance. The equivalent circuit of a supercapacitor, depicted in Figure 5a, consists of an equivalent series resistance $R_{E S R}$, followed by a constant phase element $Q$ (which models real double-layer capacitance) in parallel with a charge transfer resistance $R_{C T}$ that is in series with a linear diffusion element $M$. Nyquist plots from electrochemical impedance spectroscopy (EIS), shown in Figure 5b, can be used to understand the impact of the GO additive on the resistive components of the system. $R_{E S R}$ is measured as the intercept of the curve with the real impedance axis in the high-frequency range (small magnitudes of real and imaginary impedance). It represents the sum of the resistances associated with the electrode, electrical contacts, and electrolyte.[48,49] $R_{C T}$ is associated with the kinetics of Faradaic reactions at the electrode-electrolyte interface and is determined as the diameter of the best-fit semi-circle in the mid-frequency range of the Nyquist plot, measured along the real impedance axis.[50,51] $M$ describes the frequency-dependent, finite-length diffusion of ions in the electrolyte and contains both a pseudocapacitive and a resistive component. It behaves as a Warburg impedance at high frequencies and a $R+C$ element at low frequencies, appearing in the Nyquist plot to the right of the semicircle as an approximately $45^{\circ}$ line, followed by a near-vertical line.[52,53] The frequency at which the curve transitions from 
the semicircular to the Warburg region, where it exhibits a local minimum, is known as the knee frequency $\left(f_{k}\right)$, below which capacitive behavior begins to dominate and ions can diffuse more easily into micropores.[54] A perfect capacitor is represented by a line perpendicular to the $\mathrm{Z}_{\mathrm{Re}}$ axis in the low-frequency region.[55]

As seen in Figure 5b, the addition of $1.7 \mathrm{wt} \% \mathrm{GO}$ to RF precursors lowered $R_{E S R}$ by $24 \%$ ( $0.32 \Omega$ compared to $0.42 \Omega$ for the RFA-P). This reduction is hypothesized to be due mainly to the superior electrical conductivity of the cross-linked graphene framework of the GO-RFA-P versus the more amorphous RFA-P. Improved electrical contact between the current collector and GO-RFA-P active material compared to RFA-P is a possible secondary factor, though less likely given the similar testing conditions and supercapacitor device preparation for the two materials. The Warburg region is slightly shorter for the GO-RFA-P, spanning about $0.66 \Omega$ on the real impedance axis compared to $0.77 \Omega$ for the RFA-P, which is also indicative of less obstruction of ion movement.[48] Additionally, $R_{C T}$ was lowered by $72 \%$ ( $0.21 \Omega$ compared to $0.81 \Omega$ for the RFA-P), most likely due to the wider, more open pore structure of the GO-RFA-P as seen in Figure 2. With a greater mesoporous structure, the ions in the electrolyte can more easily access all pores in the active material, lowering the diffusionrelated resistance; also, contact area at the electrode-electrolyte interface is increased, which is consistent with the higher capacitance per unit surface area of the GO-RFA-P. Finally, the capacitive region of the GO-RFA-P is observed to not only begin at a lower impedance value than the RFA-P, but also at a frequency almost seven times higher $\left(f_{k}=3930 \mathrm{~Hz}\right.$ for GO-RFAP versus $540 \mathrm{~Hz}$ for RFA-P), indicating enhanced specific power capability of the GO-RFAP.[56] Furthermore, the steeper slope of the curve in this region for the GO-RFA-P indicates more ideal capacitive behavior and less diffusion resistance of redox species in the electrolyte during charging and discharging.[49]

Cyclic voltammetry (CV) sweeps were conducted at a rate of $20 \mathrm{mV} \mathrm{s}^{-1}$. The resulting current-voltage plot (Figure 5c) for the GO-RFA-P supercapacitor exhibits a pronounced 
rectangular shape, which indicates close to ideal electric double-layer capacitance behavior. In contrast, the voltammogram for the RFA-P supercapacitor has more rounded corners, indicating (in agreement with the EIS data) larger resistance to ion diffusion that causes the current to respond more slowly to changes in the direction of the voltage sweep.[57] Thus, in spite of the similar values of specific capacitance, the power density of the GO-RFA-P supercapacitor is superior to that of the RFA-P.

Higher resistance, as seen in both the Nyquist and CV plots, can be directly measured through the rate performance. Figure $5 \mathrm{~d}$ depicts the impact of GO on rate capability. At low current densities, RFA-P and GO-RFA-P both exhibit relatively high capacitances. However, as the rate is increased, the capacitance of the pure RFA carbons drop due to excessive resistance within the carbon. For the RFA-P, by the highest tested rate of $6.7 \mathrm{~A} \mathrm{~g}^{-1}$ the capacitance is only $68 \%$ of the capacitance at the lowest rate of $0.34 \mathrm{~A} \mathrm{~g} \mathrm{~g}^{-1}$. The graphene aerogel uniquely and dramatically increases the rate performance with $90 \%$ capacitance retention over an even wider range of current densities, $0.39 \mathrm{~A} \mathrm{~g}^{-1}$ to $7.8 \mathrm{~A} \mathrm{~g}^{-1}$. Furthermore, the GO-loaded material has $18 \%$ higher gravimetric capacitance and $62 \%$ higher areal capacitance at 7.8 $\mathrm{A} \mathrm{g} \mathrm{g}^{-1}$ versus the pure RF synthesized carbons at the slightly lower rate of 6.7 $\mathrm{A}^{-1}$. It is believed that the performance improvement can be attributed to the increase in conductive pathways for both ions and electron mobility within the carbon in the presence of pyrolyzed GO.

\subsection{Absorptive Properties of Pyrolyzed Aerogels}

The average wet-to-dry mass ratio for the GO-RFA-P in cyclohexane after 10 minutes is 7.17 , which is 1.6 times greater than that of the RFA-P (4.50). This result is made even more striking considering that the total pore volume of the former is only about half that of the latter, as evidenced by the aerogels' nitrogen sorption isotherms (Figure 2c), whose maxima are proportional to their respective pore volumes. The maximum $\mathrm{N}_{2}$ loading of the GO-RFA-P was 
$700 \mathrm{~cm}^{3} \mathrm{~g}^{-1}$ compared to $1410 \mathrm{~cm}^{3} \mathrm{~g}^{-1}$ for the RFA-P, which correspond to total pore volumes of $1.08 \mathrm{~cm}^{3} \mathrm{~g}^{-1}$ and $2.19 \mathrm{~cm}^{3} \mathrm{~g}^{-1}$ respectively. Thus, per unit pore volume, the GO-RFA-P absorbs 3.2 times as much cyclohexane as the RFA-P, in line with expectations that the graphene network in the former enhances its affinity for non-polar liquids.

\section{Conclusion}

In summary, we have presented a method for the ultrafast synthesis of graphene aerogels based on the polycondensation of resorcinol, formaldehyde, and graphene oxide with $\mathrm{HCl}$ as the catalyst and acetonitrile as the solvent. This synthetic approach greatly reduces the gelation time from many hours or even several days (using the traditional base-catalyzed route) to just 1-2 hours, making it easier to produce graphene aerogels on a large scale within reasonable time and potentially reducing their cost. Our graphene aerogels maintained the high surface area and porosity reported for plain RF-derived carbon aerogels, making them attractive candidates for applications such as supercapacitors, solar cells, catalyst supports, and oil sorption, which benefit from such characteristics as well as the intrinsic properties of graphene. As a vivid illustration of the favorable impact of graphene on supercapacitor performance, graphene aerogels synthesized by our rapid technique exceeded aerogels of plain carbonized RF in areal capacitance and power capability, suggesting that our work could accelerate the manufacture of high-performing energy storage devices. Conceivably, the capacitance of the graphene aerogels could be further improved by tailoring surface area and pore volume using $\mathrm{CO}_{2}$ activation and varying the amount of GO loading; or by experimenting with aqueous electrolytes with smaller ion size, as opposed to the organic electrolyte used in this study. We also demonstrate that our graphene aerogels hold promise as environmental sorbents, absorbing significantly more cyclohexane than plain carbonized RF aerogels over the same period of time, despite having a lower pore volume. Future research efforts in rapid graphene aerogel synthesis 
will be directed toward techniques in water, which is less expensive, safer to use, and more environmentally friendly than acetonitrile.

\section{Acknowledgements}

This research was made possible by start-up funding from the University of Washington. The authors are also grateful for support provided by the Pacific Northwest National Laboratory (PNNL) Initiative on Materials Synthesis and Simulations Across Scales $\left(\mathrm{MS}^{3}\right)$ conducted under the Laboratory Directed Research and Development Program. PNNL is operated by Battelle Memorial Institute for the U.S. DOE under contract DE-AC 06-76RLO 1830. The authors also thank E. James Davis for the donation of an optical spectrometer with an LN2cooled detector.

\section{References}

[1] Balandin AA, Ghosh S, Bao W, Calizo I, Teweldebrhan D, Miao F, et al. Superior Thermal Conductivity of Single-Layer Graphene. Nano Lett 2008;8:902-7. doi:10.1021/n10731872.

[2] Ghosh S, Calizo I, Teweldebrhan D, Pokatilov EP, Nika DL, Balandin AA, et al. Extremely high thermal conductivity of graphene: Prospects for thermal management applications in nanoelectronic circuits. Appl Phys Lett 2008;92:151911. doi:10.1063/1.2907977.

[3] Li X, Zhang G, Bai X, Sun X, Wang X, Wang E, et al. Highly conducting graphene sheets and Langmuir-Blodgett films. Nat Nanotechnol 2008;3:538-42. doi:10.1038/nnano.2008.210.

[4] El-Kady MF, Strong V, Dubin S, Kaner RB. Laser Scribing of High-Performance and Flexible Graphene-Based Electrochemical Capacitors. Science 2012;335:1326-30. doi:10.1126/science.1216744.

[5] Hwang EH, Adam S, Das Sarma S. Carrier Transport in Two-Dimensional Graphene Layers. Phys Rev Lett 2007;98:186806. doi:10.1103/PhysRevLett.98.186806.

[6] Morozov SV, Novoselov KS, Katsnelson MI, Schedin F, Elias DC, Jaszczak JA, et al. Giant Intrinsic Carrier Mobilities in Graphene and Its Bilayer. Phys Rev Lett 2008;100:016602. doi:10.1103/PhysRevLett.100.016602.

[7] Kuchta B, Firlej L, Mohammadhosseini A, Boulet P, Beckner M, Romanos J, et al. Hypothetical High-Surface-Area Carbons with Exceptional Hydrogen Storage Capacities: Open Carbon Frameworks. J Am Chem Soc 2012;134:15130-7. doi:10.1021/ja306726u.

[8] Lee G-H, Cooper RC, An SJ, Lee S, Zande A van der, Petrone N, et al. High-Strength Chemical-Vapor-Deposited Graphene and Grain Boundaries. Science 2013;340:1073-6. doi:10.1126/science.1235126. 
[9] Al-Muhtaseb SA, Ritter JA. Preparation and Properties of Resorcinol-Formaldehyde Organic and Carbon Gels. Adv Mater 2003;15:101-14. doi:10.1002/adma.200390020.

[10] Li J, Li J, Meng H, Xie S, Zhang B, Li L, et al. Ultra-light, compressible and fireresistant graphene aerogel as a highly efficient and recyclable absorbent for organic liquids. J Mater Chem A 2014;2:2934-41. doi:10.1039/C3TA14725H.

[11] Andjelkovic I, Tran DNH, Kabiri S, Azari S, Markovic M, Losic D. Graphene Aerogels Decorated with $\alpha$-FeOOH Nanoparticles for Efficient Adsorption of Arsenic from Contaminated Waters. ACS Appl Mater Interfaces 2015;7:9758-66. doi:10.1021/acsami.5b01624.

[12] Tran DNH, Kabiri S, Wang L, Losic D. Engineered graphene-nanoparticle aerogel composites for efficient removal of phosphate from water. J Mater Chem A 2015;3:6844-52. doi:10.1039/C4TA06308B.

[13] Wu Z-S, Yang S, Sun Y, Parvez K, Feng X, Müllen K. 3D Nitrogen-Doped Graphene Aerogel-Supported Fe3O4 Nanoparticles as Efficient Electrocatalysts for the Oxygen Reduction Reaction. J Am Chem Soc 2012;134:9082-5. doi:10.1021/ja3030565.

[14] Cheng W-Y, Wang C-C, Lu S-Y. Graphene aerogels as a highly efficient counter electrode material for dye-sensitized solar cells. Carbon 2013;54:291-9. doi:10.1016/j.carbon.2012.11.041.

[15] Pekala RW. Organic aerogels from the polycondensation of resorcinol with formaldehyde. J Mater Sci 1989;24:3221-7. doi:10.1007/BF01139044.

[16] Zhu Y, Hu H, Li W, Zhang X. Resorcinol-formaldehyde based porous carbon as an electrode material for supercapacitors. Carbon 2007;45:160-5. doi:10.1016/j.carbon.2006.07.010.

[17] Saliger R, Fischer U, Herta C, Fricke J. High surface area carbon aerogels for supercapacitors. J Non-Cryst Solids 1998;225:81-5. doi:10.1016/S0022-3093(98)001045.

[18] Li J, Wang X, Huang Q, Gamboa S, Sebastian PJ. Studies on preparation and performances of carbon aerogel electrodes for the application of supercapacitor. J Power Sources 2006;158:784-8. doi:10.1016/j.jpowsour.2005.09.045.

[19] Zhang W, Huang Z-H, Zhou C, Cao G, Kang F, Yang Y. Porous carbon for electrochemical capacitors prepared from a resorcinol/formaldehyde-based organic aquagel with nano-sized particles. J Mater Chem 2012;22:7158-63. doi:10.1039/C2JM16276H.

[20] Zhang K, Ang BT, Zhang LL, Zhao XS, Wu J. Pyrolyzed graphene oxide/resorcinolformaldehyde resin composites as high-performance supercapacitor electrodes. J Mater Chem 2011;21:2663. doi:10.1039/c0jm02850a.

[21] Worsley MA, Pauzauskie PJ, Olson TY, Biener J, Satcher JH, Baumann TF. Synthesis of Graphene Aerogel with High Electrical Conductivity. J Am Chem Soc 2010;132:14067-9. doi:10.1021/ja1072299.

[22] Worsley MA, Olson TY, Lee JRI, Willey TM, Nielsen MH, Roberts SK, et al. High Surface Area, sp2-Cross-Linked Three-Dimensional Graphene Monoliths. J Phys Chem Lett 2011;2:921-5. doi:10.1021/jz200223x.

[23] Mulik S, Sotiriou-Leventis C, Leventis N. Time-Efficient Acid-Catalyzed Synthesis of Resorcinol-Formaldehyde Aerogels. Chem Mater 2007;19:6138-44. doi:10.1021/cm071572m.

[24] Park S, An J, Jung I, Piner RD, An SJ, Li X, et al. Colloidal Suspensions of Highly Reduced Graphene Oxide in a Wide Variety of Organic Solvents. Nano Lett 2009;9:1593-7. doi:10.1021/n1803798y.

[25] Guo K, Song H, Chen X, Du X, Zhong L. Graphene oxide as an anti-shrinkage additive for resorcinol-formaldehyde composite aerogels. Phys Chem Chem Phys 2014;16:11603. doi:10.1039/c4cp00592a. 
[26] Aligizaki KK. Pore Structure of Cement-Based Materials: Testing, Interpretation and Requirements. CRC Press; 2005.

[27] Peigney A, Laurent C, Flahaut E, Bacsa RR, Rousset A. Specific surface area of carbon nanotubes and bundles of carbon nanotubes. Carbon 2001;39:507-14. doi:10.1016/S0008-6223(00)00155-X.

[28] Li J, Shirai T, Fuji M. Rapid carbothermal synthesis of nanostructured silicon carbide particles and whiskers from rice husk by microwave heating method. Adv Powder Technol 2013;24:838-43. doi:10.1016/j.apt.2013.02.003.

[29] Van Dijen FK, Metselaar R. The chemistry of the carbothermal synthesis of $\beta$-SiC: Reaction mechanism, reaction rate and grain growth. J Eur Ceram Soc 1991;7:177-84. doi:10.1016/0955-2219(91)90035-X.

[30] Hashimoto S, Ohashi S, Hirao K, Zhou Y, Hyuga H, Honda S, et al. Mechanism for the formation of $\mathrm{SiC}$ by carbothermal reduction reaction using a microwave heating technique. J Ceram Soc Jpn 2011;119:740-4.

[31] Seehra MS, Pavlovic AS. X-Ray diffraction, thermal expansion, electrical conductivity, and optical microscopy studies of coal-based graphites. Carbon 1993;31:557-64. doi:10.1016/0008-6223(93)90109-N.

[32] Lambert JB, Shurvell HF, Cooks RG. Introduction to Organic Spectroscopy. 1st edition. New York: Macmillan; 1987.

[33] Li W-C, Lu A-H, Guo S-C. Characterization of the microstructures of organic and carbon aerogels based upon mixed cresol-formaldehyde. Carbon 2001;39:1989-94. doi:10.1016/S0008-6223(01)00029-X.

[34] Coates J. Interpretation of Infrared Spectra, A Practial Approach. Encycl Anal Chem 2000:10815-37.

[35] Ganguly A, Sharma S, Papakonstantinou P, Hamilton J. Probing the Thermal Deoxygenation of Graphene Oxide Using High-Resolution In Situ X-ray-Based Spectroscopies. J Phys Chem C 2011;115:17009-19. doi:10.1021/jp203741y.

[36] Boukhvalov DW, Katsnelson MI. Modeling of Graphite Oxide. J Am Chem Soc 2008;130:10697-701. doi:10.1021/ja8021686.

[37] Szabó T, Berkesi O, Forgó P, Josepovits K, Sanakis Y, Petridis D, et al. Evolution of Surface Functional Groups in a Series of Progressively Oxidized Graphite Oxides. Chem Mater 2006;18:2740-9. doi:10.1021/cm060258+.

[38] Poljansek I, Krajnc M. Characterization of phenol-formaldehyde prepolymer resins by in line FT-IR spectroscopy. Acta Chim Slov 2005;52:238-44.

[39] Stuart BH. Infrared Spectroscopy: Fundamentals and Applications. 1 edition. Chichester, West Sussex, England ; Hoboken, NJ: Wiley; 2004.

[40] Malard LM, Pimenta MA, Dresselhaus G, Dresselhaus MS. Raman spectroscopy in graphene. Phys Rep 2009;473:51-87. doi:10.1016/j.physrep.2009.02.003.

[41] Sasaki K, Tokura Y, Sogawa T. The Origin of Raman D Band: Bonding and Antibonding Orbitals in Graphene. Crystals 2013;3:120-40. doi:10.3390/cryst3010120.

[42] Tuinstra F, Koenig JL. Raman Spectrum of Graphite. J Chem Phys 1970;53:1126-30. doi:10.1063/1.1674108.

[43] Wang Y, Su F, Wood CD, Lee JY, Zhao XS. Preparation and Characterization of Carbon Nanospheres as Anode Materials in Lithium-Ion Secondary Batteries. Ind Eng Chem Res 2008;47:2294-300. doi:10.1021/ie071337d.

[44] Sharma CS, Kulkarni MM, Sharma A, Madou M. Synthesis of carbon xerogel particles and fractal-like structures. Chem Eng Sci 2009;64:1536-43. doi:10.1016/j.ces.2008.12.013.

[45] Cançado LG, Takai K, Enoki T, Endo M, Kim YA, Mizusaki H, et al. General equation for the determination of the crystallite size La of nanographite by Raman spectroscopy. Appl Phys Lett 2006;88:163106. doi:10.1063/1.2196057. 
[46] Kuhn J, Brandt R, Mehling H, Petričević R, Fricke J. In situ infrared observation of the pyrolysis process of carbon aerogels. J Non-Cryst Solids 1998;225:58-63. doi:10.1016/S0022-3093(98)00009-X.

[47] Milev AS, Tran NH, Kannangara GSK, Wilson MA. Unoccupied electronic structure of ball-milled graphite. Phys Chem Chem Phys 2010;12:6685. doi:10.1039/b926345d.

[48] Stoller MD, Park S, Zhu Y, An J, Ruoff RS. Graphene-Based Ultracapacitors. Nano Lett 2008;8:3498-502. doi:10.1021/n1802558y.

[49] Xu K, Zou R, Li W, Liu Q, Wang T, Yang J, et al. Carbon-coated mesoporous NiO nanoparticles as an electrode material for high performance electrochemical capacitors. New J Chem 2013;37:4031-6. doi:10.1039/C3NJ00890H.

[50] Balakrishnan A, Subramanian KRV. Nanostructured Ceramic Oxides for Supercapacitor Applications. CRC Press; 2014.

[51] Pujadó MP. Carbon Nanotubes as Platforms for Biosensors with Electrochemical and Electronic Transduction. Springer Science \& Business Media; 2012.

[52] Gerwig R, Fuchsberger K, Schroeppel B, Link GS, Heusel G, Kraushaar U, et al. PEDOT-CNT Composite Microelectrodes for Recording and Electrostimulation Applications: Fabrication, Morphology, and Electrical Properties. Front Neuroengineering 2012;5. doi:10.3389/fneng.2012.00008.

[53] Joerg I. Physically based Impedance Modelling of Lithium-Ion Cells. KIT Scientific Publishing; 2014.

[54] Basri NH, Dolah BNM. Physical and electrochemical properties of supercapacitor electrodes derived from carbon nanotube and biomass carbon. Int J Electrochem Sci 2013;8:257-73.

[55] Wang H, Zhu B, Jiang W, Yang Y, Leow WR, Wang H, et al. A Mechanically and Electrically Self-Healing Supercapacitor. Adv Mater 2014;26:3638-43. doi:10.1002/adma.201305682.

[56] Du C, Pan N. High power density supercapacitor electrodes of carbon nanotube films by electrophoretic deposition. Nanotechnology 2006;17:5314. doi:10.1088/09574484/17/21/005.

[57] Shaijumon MM, Ou FS, Ci L, Ajayan PM. Synthesis of hybrid nanowire arrays and their application as high power supercapacitor electrodes. Chem Commun 2008:2373. doi:10.1039/b800866c. 